\title{
Categorização e Identificação Etária em uma Amostra de Idosos Brasileiros Residentes na Comunidade
}

\author{
Age Categorization and Identification in a Community-Dwelling \\ Sample of Brazilian Elderly
}

\author{
Samila Sathler Tavares Batistoni ${ }^{*}, a$, Stéfani Martins Prestes ${ }^{a}$, Meire Cachioni ${ }^{a}$, \\ Deusivânia Vieira da Silva Falcão ${ }^{a}$, Andrea Lopes ${ }^{a}$, Mônica Sanches Yassuda ${ }^{a}$ \\ \& Anita Liberalesso Neri ${ }^{b}$ \\ ${ }^{a}$ Escola de Artes, Ciências e Humanidades da Universidade de São Paulo - EACH/USP \\ ${ }^{b}$ Universidade de Campinas - UNICAMP, Campinas, SP, Brasil
}

\begin{abstract}
Resumo
Indicadores de atitudes etárias representados por categorização e identificação etária e suas relações com variáveis sociodemográficas e de saúde física e emocional foram investigados entre idosos residentes na comunidade. A amostra foi composta por 302 idosos participantes do Estudo FIBRA - Fragilidade em Idosos Brasileiros (Pólo Universidade Estadual de Campinas [Unicamp]; $M=$ 72,3 anos; $D P=5,76 ; 64,6 \%$ mulheres), com escolaridade média de 3,32 anos $(D P=2,61)$ e renda de um a três salários mínimos $(53,7 \%)$. A existência e atribuição de idade para início da velhice foram tomadas como indicadoras de categorização etária e o autorrelato de sentir-se idoso indicou identificação etária. Embora a maior parte dos idosos tenha atribuído uma idade para esse início ( $M$ $=62,3$ anos; $D P=14,66)$, a maioria $(60,3 \%)$ não se identificou como pertencente a essa categoria etária. Escolaridade, fragilidade e satisfação com a vida revelaram ser importantes preditores dessas atitudes em relação à velhice.
\end{abstract}

Palavras-chave: Atitudes, idosos, envelhecimento.

\begin{abstract}
This study investigated age categorization and identification among elderly living in the community and their relationship with sociodemographic variables and physical and emotional health. The sample consisted of 302 elderly participants in the FIBRA study - Fragilidade em Idosos Brasileiros (Polo Universidade Estadual de Campinas [Unicamp]; $M=72.3$ years old, $S D=5.76 ; 64.6 \%$ women), with average education of 3.32 years $(S D=2.61)$ and income from one to three minimum wages (53.7\%). The belief of existence and age attributed to the onset of old age were taken as indicators of age categorization and the self-report of feeling like an elderly person was taken as age identification. Although most elderly have assigned an age for the onset of old age $(M=62.3$ anos; $S D=14.66)$, most $(60.3 \%)$ are not identified as belonging to this age category. Education, frailty and life satisfaction were found to be important predictors of these attitudes towards old age.

Keywords: Attitudes, elderly, aging.
\end{abstract}

$\mathrm{O}$ estudo das atitudes e dos indicadores atitudinais em relação à velhice e ao envelhecimento são tópicos de especial relevância, principalmente entre as sociedades ocidentais, uma vez que ainda dominam modelos sociais ageístas que consideram a velhice como inerentemente

\footnotetext{
* Endereço para correspondência: Universidade de São Paulo, Escola de Artes, Ciências e Humanidades, Av. Arlindo Béttio, 1000, Ermelino Matarazzo, Sala 204 N, A1, São Paulo, SP, Brasil 03828000. E-mail: samilabatistoni@usp.br

Agradecimentos ao Conselho Nacional de Desenvolvimento Científico e Tecnológico (CNPq) pelo Financiamento do Estudo FIBRA (Polo Universidade Estadual de Campinas) e aos alunos do Curso de Gerontologia da Escola de Artes, Ciências e Humanidades da Universidade de São Paulo (EACH USP) que participaram da coleta dos dados em Ermelino Matarazzo.
}

negativa e onde indicadores positivos de saúde física são relacionados à manutenção de uma identidade etária jovem. As primeiras contribuições empíricas à temática datam de 1950 advindas da Psicologia e das Ciências Sociais americanas e inglesas cujas evidências já revelavam que características socioculturais e de experiências pessoais afetam crenças, sentimentos e comportamentos relacionados à idade, à velhice e ao envelhecimento (Barret, 2003; Batistoni \& Namba, 2010; Westerhof, Barret, \& Steverink, 2003).

Dentre os indicadores cognitivos e afetivos de tais atitudes, em especial, figuram os conceitos de categorização etária e identificação etária. Categorização etária é o processo de classificar as pessoas como pertencentes a certo grupo etário indicado pela demarcação de uma idade, de 
uma faixa etária ou por rótulos etários como "jovem" ou "velho" (Abrams \& Hogg, 2001). Por sua vez, identificação etária é o grau ou extensão que uma pessoa se identifica com uma categoria etária e, portanto, um componente do autoconceito (Tajfel, 1981; Westerhof, 2008). Tais indicadores são considerados como bases psicológicas para a formação de estereótipos e preconceitos etários trazendo implicações sobre as ações e escolhas pessoais (Sweiry \& Willitts, 2011).

As percepções individuais e experiências de envelhecimento, como traduzidas nos conceitos de categorização etária e identificação etária, constituem-se em um processo dinâmico que envolve trocas contínuas entre o self e as normas sociais (Steverink, Westerhof, Bode, \& Dittmann-Kohli, 2001). Pertencer a uma categoria etária, mas não se identificar com esta, pode revelar motivações pessoais para ganhar distinção positiva ou negativa entre os indivíduos, evidenciar o status que uma sociedade atribui a um determinado grupo etário e indicar o alcance de ações e mensagens dirigidas a tal grupo (Rowe, President, \& Kahn, 1996).

Evidências de estudos internacionais sobre a temática têm encontrado associações entre esses indicadores atitudinais de idosos e suas condições de saúde (Levy \& Myers, 2005; Levy, Slade, Kunkel, \& Kasl, 2002), seus comportamentos (Sarkisian, Prohaska, Wong, Hirsch, \& Mangione, 2005), seu bem-estar (Steverink et al., 2001; Westerhof et al., 2003) e componentes do self como autoconceito e autoestima (Sneed \& Whitbourne, 2003). Estudos norteamericanos e ingleses encontram frequentemente que "juventude subjetiva" é associada a melhores indicadores de bem-estar e satisfação com a vida (Westerhof \& Barret 2005), confiança nas próprias habilidades e competências (Schafer \& Shippee, 2010) e melhor saúde entre os idosos que assim se identificam (Demakakos, Gjonca, \& Nazroo, 2007), indicando reposta adaptativa em relação às transições etárias.

Correlatos de natureza sociodemográfica e macroestruturais também são encontrados no que se refere às atitudes em relação à velhice e envelhecimento. Barret (2003) destacou que o nível socioeconômico é um dos principais determinantes da experiência subjetiva do envelhecimento, pois influencia a forma como o indivíduo pensa sobre a sua própria idade e as fases da vida em geral. Segundo revisão da literatura realizada pelo autor, os indivíduos de menor nível socioeconômico são mais propensos a classificar-se como "velhos" ou "idosos" e sentirem-se mais velhos do que sua idade cronológica.

A esse respeito, Barret (2003) e Schafer e Shippee (2010) argumentam que as percepções do curso de vida, do tempo e da natureza das transições da vida variam de acordo com a posição social, como nível socioeconômico, gênero, raça e número de eventos estressantes de vida. Por exemplo, pessoas de nível socioeconômico mais baixo (com menor educação, renda e oportunidades ocupacionais) experimentam um padrão de desvantagem cumulativa ao longo da vida, bem como um curso de vida temporalmente mais denso na medida em que experimentam as transições importantes, como o casamento, o nascimento de filhos e início da vida de trabalho em geral, mais cedo do que os de melhor condições sociais. Esse fato tem sido encontrado em muitos estudos, porém a desvantagem relativa em relação à saúde dessas pessoas constitui-se em umas das explicações para esses achados (Diener, Suh, Lucas, \& Smith, 1999).

Abrams, Vauclair e Swift (2011) examinaram como diferenças estruturais entre os países europeus, tais como políticas, urbanização, envelhecimento demográfico e nível de bem-estar socioeconômico influenciam as atitudes etárias. Resumidamente, as evidências do estudo revelaram que: as pessoas mais velhas possuem atitudes mais favoráveis aos idosos do que as pessoas mais jovens, em especial, as mulheres; as pessoas com maior nível de educação são mais conscientes em relação ao preconceito com a idade, mas não se sentem tão fortemente afetadas por elas; aqueles que se descrevem como mais pobres revelam atitudes menos favoráveis em relação às pessoas com idade superior a 70 anos; países com maior proporção de idosos possuem visões mais positivas de pessoas com idade superior a 70 anos; populações mais urbanizadas relatam possuir menos amigos próximos com idade superior a 70 anos; países que valorizam a autonomia pessoal (tais como a independência individual) possuem atitudes mais positivas em relação aos idosos e também experimentam níveis mais baixos de preconceito etário.

Categorização e identificação etária ainda são pouco estudadas no contexto brasileiro. Dados sobre essa temática foram encontrados apenas em um estudo com grande amostra comunitária de idosos realizado pela Fundação Perseu Abramo e disponível em formato de livro (Estudo Idosos no Brasil - Vivências, Desafios e Expectativas na Terceira Idade; Neri, 2007b) que incluiu em seu protocolo de pesquisa as seguinte questões: " $\mathrm{O}$ (a) senhor(a) se sente idoso?" e "Em que idade começa a velhice?". O estudo contou com a participação de 2.136 idosos, em 204 municípios nas cinco regiões do país. Nesse estudo, grande parte relatou não se sentir idoso (53\%). A maioria dos idosos relatou que a velhice começa após os 60 anos (58\%), e as mulheres pesquisadas relataram se sentir menos idosas do que os homens. Entretanto, a percepção da chegada da velhice pelos entrevistados conjugou-se com aspectos negativos como o enfrentamento de doenças nessa etapa da vida.

Uma vez que a literatura internacional salienta que as avaliações subjetivas que os indivíduos fazem a respeito da sua própria idade influenciam o bem-estar (Westerhof \& Barret, 2005), a motivação e os investimentos em recursos socioemocionais (Carstensen, 2006) e afetam condições de saúde física e mental (Demakakos et al., 2007), o levantamento de dados contextualizados sobre a temática podem trazer implicações para o conhecimento e para as práticas em Psicologia e Gerontologia no Brasil. 
Batistoni, S. S. T., Prestes, S. M., Cachioni, M., Falcão, D. V. S., Lopes, A., Yassuda, M. S. \& Neri, A. L. (2015). Categorização e Identificação Etária em uma Amostra de Idosos Brasileiros Residentes na Comunidade.

Nesse sentido, o presente estudo examinou, no contexto de idosos comunitários brasileiros, as atitudes em relação à velhice e ao envelhecimento por meio dos indicadores de categorização e identificação etária. Complementarmente, visou identificar relações desses indicadores com variáveis sociodemográficas e de condições de saúde física e emocional.

\section{Método}

O presente estudo é parte integrante dos esforços de pesquisa da Rede FIBRA (Rede para o Estudo da Fragilidade em Idosos Brasileiros), dedicada a identificar condições de fragilidade em idosos urbanos residentes na comunidade, com 65 anos e mais, e suas relações com variáveis demográficas, socioeconômicas, psicossociais e de saúde física (Neri et al., 2013).

Essa rede foi composta por quatro universidades públicas brasileiras (Unicamp; Universidade de São Paulo em Ribeirão Preto [USP-RP]; Universidade Federal de Minas Gerais [UFMG], e Universidade Estadual do Rio de Janeiro [UERJ]), contempladas pelo edital 17/2006, linha 2, Gerontologia clínica, do Conselho Nacional de Pesquisa (CNPq). Cada pólo nucleou os trabalhos de um grupo de instituições parceiras e, além de um protocolo de pesquisa compartilhado, teve a liberdade de planejar estudos adicionais com objetivos, definições, justificativas e delineamentos específicos.

Os dados do presente artigo foram derivados do estudo desenvolvido pela rede FIBRA, Pólo Unicamp, no Distrito de Ermelino Matarazzo, São Paulo (SP) em parceria com o Curso de Graduação em Gerontologia da Escola de Artes, Ciências e Humanidades da Universidade de São Paulo (EACH/USP) e com a aprovação do Comitê de Ética em Pesquisa com Seres Humanos da Faculdade de Ciências Médicas/Unicamp, mediante o parecer 208/2007.

Localizado na região leste do município de São Paulo, o subdistrito de Ermelino Matarazzo possui uma população de cerca de 106.000 habitantes e baixo status socioeconômico. Os idosos ( $\geq 65$ anos) representam 4,5\% dos residentes no distrito (Fundação Sistema Estadual de Análise de Dados [Seade], 2010). Para o recrutamento dos idosos realizou-se uma amostragem aleatória simples de setores censitários urbanos do subdistrito de Ermelino Matarazzo a ser visitados por duplas recrutadores treinados. O número de setores visitados (62) correspondeu à razão entre o número de idosos pretendidos e o número total de setores. Os idosos a ser identificados deveriam ter idade acima de 65 anos, compreender as instruções, aceitar participar e ser um residente fixo no endereço. Os critérios de exclusão foram (a) presença de comprometimento cognitivo severo sugestivo de demência autorrelatado por familiares ou cuidadores no recrutamento ou investigado por meio da aplicação do Mini Exame do Estado Mental (MEEM; Folstein, Folstein, \& McHugh, 1975) no momento da coleta, (b) ser cadeirante ou aca- mado, (c) ter sequelas de acidente vascular encefálico com perda localizada de força e/ou afasia, (d) Doença de Parkinson sem tratamento, (e) déficits severos em audição ou visão que impedem a comunicação, e (f) ser doente terminal. Os critérios de inclusão e exclusão foram adaptados do Cardiovascular Health Study (CHS), descritos por Ferrucci e colaboradores (2004). Após identificação dos idosos e aceitação em participar da pesquisa, os participantes assinaram o Termo de Consentimento Livre e Esclarecido e foram entrevistados, em data próxima, em centros comunitários (igrejas ou centros de convivência social) próximos às suas residências por entrevistadores treinados provenientes do Curso de Graduação em Gerontologia da EACH/USP. As sessões de entrevista duraram de 40 a 120 minutos.

\section{Participantes}

Trezentos e dois idosos compuseram a amostra de Ermelino Matarazzo (SP).

\section{Instrumentos}

Selecionou-se do protocolo geral do FIBRA Ermelino Matarazzo as seguintes variáveis e instrumentos:

1. Variáveis sociodemográficas: Idade, gênero, estado civil, escolaridade e renda pessoal.

2. Identificação etária: avaliada por meio da questão "Você se sente idoso?", cujas possibilidades de resposta foram: (1) sim; (2) não; (3) às vezes.

3. Categorização etária: avaliada por meio da questão "Existe idade para o começo da velhice?" cujas possibilidades de resposta foram (1) sim; (2) não. Para os indivíduos que responderam (1) sim, foi feita uma pergunta complementar em formato aberto: "Qual a idade para o começo da velhice?"

4. Depressão: avaliada através da Escala de Depressão Geriátrica (GDS-15; Almeida \& Almeida, 1999; Sheikh \& Yesavage, 1986). Trata-se de uma escala de rastreio de sintomatologia depressiva indicada pelo alcance de 6 ou mais pontos.

5. Perfis de Fragilidade: definidos a partir do fenótipo de fragilidade proposto por Fried e colaboradores (2001), medidos por (a) autorrelato de perda de peso não intencional no último ano, igual ou superior a $4,5 \mathrm{~kg}$ ou a $5 \%$ do peso corporal; (b) fadiga indicada pelas respostas sempre ou quase sempre a dois itens da Center for Epidemiological Study - Depression (CES-D) que perguntam sobre queda percebida no nível de energia na última semana; (c) baixa taxa de gasto calórico semanal em exercícios físicos e em atividades domésticas autorrelatados - pontuam para este critério aqueles cuja taxa de gasto calórico em kcal localiza-se abaixo do $1^{\circ}$ quintil da amostra (com ajuste por sexo); (d) baixa força de preensão, quando a média em $\mathrm{kg}_{\mathrm{f}}$ de três medidas consecutivas tomadas com dinamômetro hidráulico manual localiza-se abaixo do $1^{\circ}$ quintil da amostra (com ajustes 
por IMC e sexo); (e) lentidão da marcha, quando a média de três medidas consecutivas do tempo (em segundos) para percorrer $4,6 \mathrm{~m}$ no plano e em passo usual, é superior ao percentil 80 da amostra (as médias são ajustadas por sexo e altura). Pontuação em três ou mais critérios indica fragilidade, em um ou dois, pré-fragilidade e, em nenhum, não-fragilidade (Fried et al., 2001)

6. Satisfação geral com a vida e satisfação com a vida comparada a outros - avaliada por meio das seguintes questões: " $O(a)$ senhor(a) está satisfeito com sua vida?" "Em comparação à outras pessoas da sua idade, quão satisfeito o senhor(a) está com sua vida?" As possibilidades de respostas foram: (1) Pouco satisfeito; (2) Mais ou menos satisfeito; (3) Muito satisfeito.

7. Saúde autorrelatada - avaliada por meio da seguinte questão: "Como o(a) senhor(a) sente que está a sua saúde?" As possibilidades de respostas foram: (1) muito ruim; (2) regular; (3) boa; (4) muito boa; (5) excelente.

\section{Análise dos Dados}

O perfil da amostra foi descrito por meio de indicadores de frequência e posição. Para comparação das variáveis categóricas foram utilizados os testes Qui-Quadrado. Para estudar os fatores associados a sentir-se idoso, existência de idade para a velhice e idade atribuída ao início da velhice foi utilizada a análise de regressão logística uni- variada e multivariada, com critério Stepwise de seleção de variáveis.

\section{Resultados}

A Tabela 1 descreve a amostra segundo todas as variáveis do estudo. Houve predominância do sexo feminino e idade média de 72,3 anos $(D P=5,76)$, com variação entre 65 e 92 anos. A escolaridade média entre os participantes foi de 3,42 anos $(D P=2,82)$, com variação entre 0 a 21 anos. Ser casado, ter baixa escolaridade e possuir renda familiar entre um a três salários mínimos também foi mais frequente na amostra. A maior parte dos idosos avaliou sua saúde como boa $(40,5 \%)$ ou regular $(38,5 \%)$. Mais da metade da amostra revelou um perfil de pré-fragilidade e $6,3 \%$ foram classificados como frágeis. A prevalência de depressão estimada pela GDS- 15 foi de $20,8 \%$ e, no que se referiu à satisfação geral com a vida, cerca de $66 \%$ relatou estar muito satisfeita, relato este que tem sua frequência aumentada quando os idosos comparam-se a outras pessoas da mesma idade (74,4\%).

Quanto aos critérios de identificação etária e categorização etária observou-se que a maioria dos idosos relatou não se sentir pertencente à categoria "idosos" $(60,3 \%)$. A maior parte também acredita que a velhice tem uma idade para começar $(57,5 \%)$ a qual se iniciaria em média aos 62,3 anos $(D P=14,6)$. A atribuição de idade para o início da velhice variou de 30 a 120 anos, cuja distribuição pôde ser divida em tercis: $31,2 \%$ atribuiu início antes dos 60 anos; $31,2 \%$ aos 60 anos; e 37,8\% após os 60 anos.

Tabela 1

Descrição da Amostra segundo Critérios Sociodemográficos e demais Variáveis do Estudo (N=302)

$$
n(\%)
$$

\begin{tabular}{lc}
\hline Gênero & \\
Feminino & $195(64,6)$ \\
Masculino & $107(35,4)$ \\
Idade & \\
65-69 anos & $123(40,7)$ \\
$70-74$ anos & $96(31,8)$ \\
$75-79$ anos & $49(16,2)$ \\
$\geq 80$ anos & $34(11,3)$ \\
Estado Civil & \\
Casado & $155(51,3)$ \\
Solteiro & $20(6,6)$ \\
Divorciado & $23(7,6)$ \\
Viúvo & $104(34,4)$ \\
Escolaridade & \\
0 & $69(18)$ \\
$1-4$ anos & $236(61,6)$ \\
$5-8$ anos & $64(16,7)$ \\
$\geq 9$ anos & $14(3,7)$
\end{tabular}


Batistoni, S. S. T., Prestes, S. M., Cachioni, M., Falcão, D. V. S., Lopes, A., Yassuda, M. S. \& Neri, A. L. (2015). Categorização e Identificação Etária em uma Amostra de Idosos Brasileiros Residentes na Comunidade.

Faixa de Renda

$$
\begin{aligned}
& \leq 1 \mathrm{SM} \\
& 1,1-3 \mathrm{SM} \\
& 3,1-5 \mathrm{SM} \\
& \geq 5,1 \mathrm{SM}
\end{aligned}
$$

Autoavaliação de saúde

Muito ruim

$25(8,3)$

Regular

$116(38,5)$

Boa

$122(40,5)$

Muito boa

Excelente

Fragilidade

Não-frágil

$119(39,4)$

Pré-frágil

$164(54,3)$

Frágil

$19(6,3)$

Depressão

Sim

Satisfação com a vida

Pouco satisfeito

$46(15,2)$

Mais ou menos

$57(18,9)$

Muito satisfeito

$199(65,9)$

Satisfação com a vida comparada a outros

Pouco satisfeito

$37(12,3)$

Mais ou menos

$40(13,3)$

Muito satisfeito

$224(74,4)$

Sente-se idoso

Sim

$106(35,1)$

Não

$182(60,3)$

Às vezes

Existência de idade para a velhice

Sim

$173(57,5)$

Não

$128(42,5)$

Início da velhice

$<60$ anos

$54(31,2)$

60 anos

$54(31,2)$

$>60$ anos

$65(37,8)$

As comparações entre identificação etária e categorização etária e os grupos sociodemográficos e por critérios de saúde física e emocional são apresentadas na Tabela 2. Houve diferença significativa quanto a sentir-se idoso para as variáveis: gênero (maior frequência entre as mulheres), idade (maior frequência entre os de maior idade), escolaridade (maior frequência entre os analfabetos), saúde percebida (maior frequência entre os de pior saúde), fragilidade (maior frequência entre os frágeis), depressão (maior frequência entre os com depressão) e satisfação com a vida comparada (maior frequência entre os com menor satisfação). Verificou-se diferença significativa quanto à existência de idade para a velhice para as variáveis: escolaridade (maior frequência entre os analfabetos), fragilidade (maior frequência entre os pré-frágeis), depressão (maior frequência entre os com depressão), satisfação geral com a vida e comparada a outros (maior frequência entre os mais ou menos satisfeitos). Destaca-se, entretanto, que no que se referiu à atribuição de idade para o início da velhice, os dados não revelaram diferenças significativas entre os grupos formados por critérios sociodemográficos ou de saúde física e emocional.

A Tabela 3 apresenta os resultados das análises de regressão logística univariada para identificar preditores de identificação etária. "Sentir-se idoso" foi relacionado às variáveis: satisfação com vida comparada a outros (mais ou menos satisfeito), idade (maior de 80 anos), gênero (feminino) e escolaridade (analfabetos), presença de depressão e saúde física autorrelatada (regular ou muito ruim). 
Tabela 2

Distribuição das Variáveis Sociodemográficas e de Saúde Física e Emocional segundo Identificação Etária e Categorização Etária

\begin{tabular}{cccccc}
\multicolumn{2}{c}{ Sente-se idoso Não sente-se } & Significância & Existe idade & Não existe & Significância \\
$n(\%)$ & idoso & estatística & para a velhice & idade para a & estatística \\
& $n(\%)$ & & $n(\%)$ & velhice \\
& & & & $n(\%)$
\end{tabular}

Gênero

$\begin{array}{lcccccc}\text { Feminino } & \underline{87(72,5)} & 111(61) & \mathrm{x}^{2}=4,25 ; g l=1 & 105(60,7) & 91(71,1) & \mathrm{x}^{2}=3,50 ; g l=1 \\ \text { Masculino } & 33(27,5) & \underline{71(39)} & \underline{p=0,039} & 68(39,3) & 37(28,9) & p=0,061\end{array}$

Idade

$\begin{array}{lcccccc}65-69 \text { anos } & 43(35,8) & \underline{79(43,4)} & \mathrm{x}^{2}=10,25 ; g l=3 & 63(36,4) & 59(46,1) & \mathrm{x}^{2}=3,75 ; g l=1 \\ 70-74 \text { anos } & 38(31,7) & 61(33,6) & \underline{p=0,017} & 58(33,5) & 41(32) & p=0,061 \\ 75-79 \text { anos } & 18(15) & 31(17) & & 32(18,5) & 16(12,5) & \\ \geq 80 \text { anos } & \underline{21(17,5)} & 11(6) & & 20(11,6) & 12(9,4) & \end{array}$

Escolaridade

0

1-4 anos

$\underline{30(25,2)}$

$75(63)$

$\begin{array}{cc}24(13,2) & \mathrm{x}^{2}=9,70 ; g l=2 \\ 119(65,4) & \underline{p=0,008}\end{array}$

$\underline{44(25,6)}$

$103(59,9)$

\begin{tabular}{cc}
$9(7)$ & $\mathrm{x}^{2}=17,95 ; g l$ \\
$\underline{91(71,1)}$ & $=2$ \\
$\underline{28(21,9)}$ & $p<0,001$ \\
\hline
\end{tabular}

$\geq 5$ anos

$14(11,8) \quad \underline{39(21,4)}$

$25(14,5) \quad \underline{28(21,9)}$

Renda

$\leq 1 \mathrm{SM}$

$1,1-3,0 \mathrm{SM}$

$48(41,7)$

$65(37,1)$

$\mathrm{x}^{2}=3,80 ; g l=2$

$67(40,1)$

$45(36,9)$

$\mathrm{x}^{2}=3,38 ; g l=2$

$54(47) \quad 75(42,9)$

$78(46,7)$

$51(41,8)$

$p=0,184$

$\geq 3 \mathrm{SM}$

$13(11,3) \quad 35(20)$

$22(13,2) \quad 26(21,3)$

Autoavaliação de saúde

Muito ruim

Regular

$13(11)$

$12(6,7)$

$\mathrm{x}^{2}=11,60 ; g l=4$

17 (10)

$63(37,1)$

$8(6,4)$

$73(42,9)$

$51(40,5)$

$\mathrm{x}^{2}=7,57 ; g l$

$40(33,6)$

$\underline{80(44,9)}$

Muito boa

$3(2,5)$

$\underline{14(7,9)}$

$5(2,9)$

$46(36,5)$

$12(7,1)$

$12(9,5)$

$7(5,9) \quad \underline{14} \quad(7,9)$

$9(7,1)$

Fragilidade

Não-frágil

Pré-frágil

$52(43,3)$
$56(46,7)$

$72(40)$

$\mathrm{x}^{2}=6,90 ; g l=2$

$61(35,5)$

$\underline{63(49,6)} \quad \mathrm{x}^{2}=6,0 ; g l=2$

$56(46,7) \quad \underline{102(56,7)}$

$\underline{100(58,1)}$

$57(44,9)$

$p=0,048$

Frágil

$6(3,3)$

$11(6,4)$

$7(5,5)$

Depressão

Sim

Não

$\underline{36(30)}$

$27(14,9)$

$84(70)$

$154(85,1)$

$\mathrm{x}^{2}=9,90 ; g l=1$

$\underline{42(24,4)}$

$19(14,8) \quad \mathrm{x}^{2}=4,15 ; g l=1$

Satisfação com a vida

Pouco satisfeito

Mais ou menos

Muito satisfeito

$20(16,8)$

$24(13,2)$

$30(16,6)$

$27(22,7)$

$127(70,2)$

$\mathrm{x}^{2}=3,05 ; g l=2$

$p=0,219$

$21(12,2)$

$\underline{41(23,8)}$

$110(64)$

$\underline{24(18,9)}$

$16(12,6)$

$\underline{87(68,5)}$

Satisfação com a vida comparada a outros

Pouco satisfeito

Mais ou menos

$12(10,2)$

$24(13,3)$

$\mathrm{x}^{2}=18,05 ; g l=2$

$14(8,2)$

$\underline{23(18,1)} \quad x^{2}=12,41 ; g l=2$

$\underline{28(23,7)}$

$12(6,6)$

$78(66,1)$

$\underline{145(80,1)}$

$\underline{31(18,1)}$

$9(7,1)$

$95(74,8)$

$p=0,002$

Muito satisfeito

Nota. Os valores sublinhados referem-se aos grupos que mostraram diferenças estatisticamente significativas. 
Batistoni, S. S. T., Prestes, S. M., Cachioni, M., Falcão, D. V. S., Lopes, A., Yassuda, M. S. \& Neri, A. L. (2015). Categorização e Identificação Etária em uma Amostra de Idosos Brasileiros Residentes na Comunidade.

Tabela 3

Resultados da Análise de Regressão Logística Univariada para Identificação Etária ("sentir-se idoso”)

\begin{tabular}{|c|c|c|c|c|}
\hline & Categorias & Valor- $P$ & $\mathrm{OR}^{*}$ & IC $95 \%$ OR* \\
\hline \multirow{4}{*}{ Idade } & 65-69 anos (ref.) & --- & 1,00 & --- \\
\hline & 70-74 anos & 0,630 & 1,14 & $0,66-1,98$ \\
\hline & 75-79 anos & 0,854 & 1,07 & $0,54-2,13$ \\
\hline & $\geq 80$ anos & $\underline{0,003}$ & 3,51 & $1,55-7,95$ \\
\hline \multirow{2}{*}{ Sexo } & Masculino (ref.) & --- & 1,00 & --- \\
\hline & Feminino & $\underline{0,040}$ & 1,69 & $1,02-2,78$ \\
\hline \multirow{3}{*}{ Escolaridade } & $\geq 5$ anos (ref.) & --- & 1,00 & --- \\
\hline & $1-4$ anos & 0,103 & 1,76 & $0,89-3,45$ \\
\hline & 0 ano & $\underline{0,003}$ & 3,48 & $1,54-7,85$ \\
\hline \multirow{3}{*}{ Renda pessoal } & $\leq 1.0 \mathrm{SM}$ (ref.) & --- & 1,00 & --- \\
\hline & $1.1-3.0 \mathrm{SM}$ & 0,074 & 1,94 & $0,94-4,01$ \\
\hline & $>3.0 \mathrm{SM}$ & 0,068 & 1,99 & $0,95-4,16$ \\
\hline \multirow{3}{*}{ Saúde autorrelatada } & Excelente/Muito boa (ref.) & --- & 1,00 & --- \\
\hline & Boa & 0,419 & 1,40 & $0,62-3,17$ \\
\hline & Regular/Muito ruim & $\underline{0,012}$ & 2,76 & $1,25-6,11$ \\
\hline \multirow{3}{*}{ Fragilidade } & Não-frágil (ref.) & --- & 1,00 & --- \\
\hline & Pré-frágil & 0,266 & 0,76 & $0,47-1,23$ \\
\hline & Frágil & 0,056 & 2,77 & $0,98-7,86$ \\
\hline \multirow{2}{*}{ Depressão } & Não (ref.) & --- & 1,00 & --- \\
\hline & Sim & $\underline{0,002}$ & 2,44 & $1,39-4,30$ \\
\hline \multirow{3}{*}{ Satisfação com a vida } & Muito (ref.) & --- & 1,00 & --- \\
\hline & Mais ou menos & 0,128 & 1.59 & $0,88-2,88$ \\
\hline & Pouco & 0,253 & 1.47 & $0,76-2,84$ \\
\hline \multirow{3}{*}{$\begin{array}{l}\text { Satisfação com a vida } \\
\text { comparada }\end{array}$} & Muito (ref.) & --- & 1,00 & --- \\
\hline & Mais ou menos & $\leq 0,001$ & 4,34 & $2,09-9,00$ \\
\hline & Pouco & 0,848 & 0,93 & $0,44-1,96$ \\
\hline
\end{tabular}

Notas. Os valores sublinhados referem-se aos grupos que mostraram diferenças estatisticamente significativas. *OR $($ Odds Ratio $)=$ Razão de risco para sente-se idoso; ( $n=182$ não se sente idoso e $n=120$ sente-se idoso). IC $95 \%$ OR = Intervalo de $95 \%$ de confiança para a razão de risco. Ref.: nível de referência.

Por sua vez, na Tabela 4, são apresentadas as probabilidades representadas pelas variáveis do estudo para a "existência de idade para a velhice". Foram significativas: a escolaridade (analfabetos), satisfação com a vida comparada a outros (mais ou menos satisfeitos), satisfação geral com a vida (mais ou menos satisfeitos), destacando-se também as probabilidades representadas por presença de depressão e pré-fragilidade.

$\mathrm{Na}$ análise de regressão multivariada, o perfil dos indivíduos que relataram sentir-se idosos foi dado pelas variáveis: estar mais ou menos satisfeito com a vida quando comparado a outros (probabilidade 4,8 vezes maior que os muito satisfeitos) e os com menor escolaridade (probabilidade 2,2 vezes e 3,6 vezes maior para os com 1-4 anos e 0 ano em relação aos com 5 ou mais anos). $O$ perfil dos indivíduos com maior probabilidade de referir "existência de idade para velhice" foi: os com menor escolaridade (probabilidade de 6,3 vezes maior para os analfabetos em relação aos com 5 ou mais anos de estudo), os do sexo masculino (probabilidade 1,9 vez maior que os do sexo feminino), os mais ou menos satisfeitos (probabilidade 2,6 vezes maior que os muito satisfeitos), e os pré-frágeis (probabilidade 1,9 vez maior que os não-frágeis).

\section{Discussão}

O presente estudo examinou a temática da categorização e identificação etária entre idosos comunitários brasileiros e identificou relações com variáveis sociodemográficas e de saúde física e emocional.

No que se referiu à categorização etária, percebeu-se que a maior parte dos entrevistados acredita existir uma 
Tabela 4

Resultados da Análise de Regressão Logística Univariada para Categorização Etária ("existência de idade para a velhice")

\begin{tabular}{|c|c|c|c|c|}
\hline & Categorias & Valor- $P$ & $\mathrm{OR}^{*}$ & IC $95 \%$ OR* \\
\hline Idade & $\begin{array}{c}\text { 65-69 anos (ref.) } \\
70-74 \text { anos } \\
75-79 \text { anos } \\
\geq 80 \text { anos }\end{array}$ & $\begin{array}{c}--- \\
0,303 \\
0,078 \\
0,275\end{array}$ & $\begin{array}{l}1,00 \\
1,33 \\
1,87 \\
1,56\end{array}$ & $\begin{array}{c}--- \\
0,78-2,26 \\
0,93-3,76 \\
0,70-3,47\end{array}$ \\
\hline Sexo & $\begin{array}{l}\text { Feminino (ref.) } \\
\text { Masculino }\end{array}$ & $\begin{array}{c}--- \\
0,062\end{array}$ & $\begin{array}{l}1,00 \\
1,59\end{array}$ & $0,98-2,60$ \\
\hline Escolaridade & $\begin{array}{c}\geq 5 \text { anos (ref.) } \\
1-4 \text { anos } \\
0 \text { ano }\end{array}$ & $\begin{array}{c}--- \\
0,445 \\
<0,001 \\
\end{array}$ & $\begin{array}{l}1,00 \\
1,27 \\
5,48\end{array}$ & $\begin{array}{c}--- \\
0,69-2,33 \\
2,23-13,43\end{array}$ \\
\hline Renda pessoal & $\begin{array}{c}\leq 1,0 \mathrm{SM} \text { (ref.) } \\
1,1-3,0 \mathrm{SM} \\
>3,0 \mathrm{SM}\end{array}$ & $\begin{array}{c}--- \\
0,083 \\
0,104\end{array}$ & $\begin{array}{l}1,00 \\
1,81 \\
1,76\end{array}$ & $\begin{array}{c}--- \\
0,93-3,53 \\
0,89-3,48\end{array}$ \\
\hline Saúde autorrelatada & $\begin{array}{l}\text { Excelente/Muito boa } \\
\text { (ref.) } \\
\text { Boa } \\
\text { Regular/Muito ruim }\end{array}$ & $\begin{array}{c}--- \\
0,074 \\
0,162\end{array}$ & $\begin{array}{l}1,00 \\
1,96 \\
1,68\end{array}$ & $\begin{array}{c}--- \\
0,94-4,10 \\
0,81-3,45\end{array}$ \\
\hline Fragilidade & $\begin{array}{l}\text { Não-frágil (ref.) } \\
\text { Pré-frágil } \\
\text { Frágil }\end{array}$ & $\frac{0,015}{0,348}$ & $\begin{array}{l}1,00 \\
1,81 \\
1,62\end{array}$ & $\begin{array}{c}--- \\
1,12-2,93 \\
0,59-4,46\end{array}$ \\
\hline Depressão & $\begin{array}{l}\text { Não (ref.) } \\
\quad \text { Sim }\end{array}$ & $\underline{---}$ & $\begin{array}{l}1,00 \\
1,85\end{array}$ & $1,02-3,3$ \\
\hline Satisfação com a vida & $\begin{array}{l}\text { Muito (ref.) } \\
\text { Mais ou menos } \\
\text { Pouco }\end{array}$ & $\frac{0,031}{0,267}$ & $\begin{array}{l}1,00 \\
2,03 \\
0,69\end{array}$ & $\begin{array}{c}--- \\
1,07-3,85 \\
0,36-1,33\end{array}$ \\
\hline $\begin{array}{l}\text { Satisfação com a vida } \\
\text { comparada }\end{array}$ & $\begin{array}{l}\text { Muito (ref.) } \\
\text { Mais ou menos } \\
\text { Pouco }\end{array}$ & 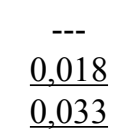 & $\begin{array}{l}1,00 \\
2,60 \\
0,46\end{array}$ & $\begin{array}{c}--- \\
1,18-5,71 \\
0,22-0,94\end{array}$ \\
\hline
\end{tabular}

Notas. Os valores sublinhados referem-se aos grupos que mostraram diferenças estatisticamente significativas. * OR $($ Odds Ratio $)=$ Razão de risco para existe idade; ( $n=128$ não existe idade e $n=173$ existe idade). IC $95 \%$ OR $=$ Intervalo de $95 \%$ de confiança para a razão de risco. Ref.: nível de referência.

idade para o início da velhice a qual, em média, é demarcada por volta dos 62 anos. Tal idade média assemelha-se à adotada no Brasil como marco etário para o usufruto de direitos e para o advento da aposentadoria no país. $\mathrm{O}$ estudo realizado pela Fundação Perseu Abramo também identificou que para a maioria dos idosos a velhice se inicia após os 60 anos. Em países europeus, o estudo de Abrams e colaboradores (2011) sobre categorização etária também encontrou uma idade semelhante para o início da velhice (63 anos). Entretanto, no presente estudo, percebeu-se uma grande variabilidade na idade atribuída ao início da velhice, variando dos 30 aos 120 anos. Chamou a atenção o fato de aproximadamente um terço da amostra acreditar que a velhice se inicia antes dos 60 anos, o que talvez reflita a influência de fatores psicossociais e culturais presentes na amostra estudada. Como ressaltado por Schafer e Shippee (2010), entre populações mais desfavorecidas economicamente, o acúmulo de desvantagens ao longo da vida ou a ocorrência de múltiplos eventos de vida estressantes influenciam a percepção de tempo vivido e do senso individual e social de envelhecimento.

A existência de uma idade específica para o início da velhice no presente estudo relacionou-se com escolaridade e indicadores de saúde física e emocional. Indivíduos com menor escolaridade se diferenciaram dos demais e apresentaram maior probabilidade de atribuir uma idade à velhice. Os idosos com perfil de pré-fragilidade, presença de depressão e uma moderada percepção de satisfação 
Batistoni, S. S. T., Prestes, S. M., Cachioni, M., Falcão, D. V. S., Lopes, A., Yassuda, M. S. \& Neri, A. L. (2015). Categorização e Identificação Etária em uma Amostra de Idosos Brasileiros Residentes na Comunidade.

Tabela 5

Resultados das Análises de Regressão Logística Multivariada para "Sentir-se Idoso"(n=279) e para "Existência de Idade para a Velhice" (n=278)

\begin{tabular}{lcccc}
\hline \multicolumn{5}{c}{ Sente-se idoso } \\
\hline & Categorias & Valor- $P$ & OR* & IC 95\% OR* \\
\hline \multirow{2}{*}{$\begin{array}{l}\text { 1. Satisfação com a vida } \\
\text { comparada }\end{array}$} & Muito (ref.) & --- & 1,00 & --- \\
& Mais ou menos & $\underline{-0,001}$ & 4,75 & $2,18-10,35$ \\
& Pouco & 0,977 & 0,99 & $0,45-2,16$ \\
2. Escolaridade & $\geq 5$ anos (ref.) & --- & 1,00 & --- \\
& $1-4$ anos & $\underline{0,035}$ & 2,22 & $1,06-4,66$ \\
& 0 ano & $\underline{0,004}$ & 3,61 & $1,52-8,5$ \\
\hline
\end{tabular}

Existência de idade para a velhice

\begin{tabular}{lcccc}
\hline & Categorias & Valor- $P$ & OR** & IC 95\% OR** \\
\hline \multirow{3}{*}{ 1. Escolaridade } & $\geq 5$ anos (ref.) & --- & 1,00 & --- \\
& $1-4$ anos & 0,182 & 1,58 & $0,81-3,07$ \\
2. Sexo & 0 ano & $\leq 0,001$ & 6,29 & $2,47-16,01$ \\
& Feminino (ref.) & --- & 1,00 & --- \\
3. Satisfação com a vida & Masculino & $\underline{0,020}$ & 1,90 & $1,11-3,28$ \\
comparada & Muito (ref.) & --- & 1,00 & --- \\
& Mais ou menos & $\underline{0,035}$ & 2,60 & $1,07-6,31$ \\
& Pouco & 0,069 & 0,48 & $0,22-1,06$ \\
4. Fragilidade & Não-frágil (ref.) & --- & 1,00 & --- \\
& Pré-frágil & $\underline{0,019}$ & 1,90 & $1,11-3,26$ \\
& Frágil & 0,710 & 0,79 & $0,23-2,72$
\end{tabular}

Notas. Os valores sublinhados referem-se aos grupos que mostraram diferenças estatisticamente significativas. *OR (Odds Ratio) = Razão de risco para "sente-se idoso"; ( $n=169$ não se sente idoso e $n=110$ sente-se idoso). IC 95\% OR = Intervalo de $95 \%$ de confiança para a razão de risco. Critério Stepwise de seleção de variáveis. **OR (Odds Ratio) = Razão de risco para existência de idade para a velhice; ( $n=120$ não existe idade e $n=158$ existe idade). IC 95\% OR = Intervalo de 95\% de confiança para a razão de risco. Critério Stepwise de seleção de variáveis.

com a vida em geral e comparada a outros, foram atributos mais frequentes entre os que acreditam existir idade para a velhice. Idosos em condições mais desvantajosas geralmente experienciam transições de vida mais cedo e talvez tenham maior propensão em identificar marcos específicos para estas (Barret, 2003; Diener et al., 1999; Schafer \& Shippee, 2010).

No que se referiu à identificação etária, a maior parte dos entrevistados relatou não se sentir idosa, ou seja, não se identificam como pertencentes a essa categoria. Dados de estudos brasileiros já apontaram que a concepção de velhice ainda permanece atrelada a aspectos negativos como perdas e incidência de doenças físicas (Neri, 2007a). A não identificação com a velhice ou a atribuição a si mesmo de uma idade subjetiva menor do que a própria idade cronológica em países ocidentais tem sido considerada pela literatura gerontológica como um indicador de bem-estar e como estratégia de autorregulação do self (Sneed \& Whitbourne, 2003). Um dos dados clássicos na literatura gerontológica é de que os indivíduos, após a meia-idade, tendem a atribuir a si mesmos uma idade subjetiva 20\% menor que sua idade cronológica (Schafer \& Shippee, 2010).

Entre os que referiram se sentir idosos houve maior frequência de mulheres. Alguns autores apontam que a conjunção de ser mulher e ser idosa, em países ocidentais, gera um duplo padrão de desvantagem associada ao envelhecimento, sendo encontrado, entre essas, maior número de queixas, depressão e insatisfação em geral quando comparado aos homens (Neri, 2007a).

Idade e escolaridade também se relacionaram com sentir-se idoso. Os indivíduos mais velhos da amostra foram mais presentes nessa categoria, talvez pelo fato de experimentarem mais frequentemente, limitações 
provenientes do envelhecimento físico e social do que os idosos mais jovens, aproximando-se do conceito de velhice compartilhado socialmente. Escolaridade novamente revelou-se uma variável importante no estudo das atitudes em relação à velhice. A maior parte dos analfabetos descreveu-se como idoso e menor escolaridade representou maior probabilidade para sentir-se idoso. Autores argumentam que baixo nível de escolaridade resume ou carrega consigo outras influências que talvez incidam sobre a percepção da própria idade e do processo de envelhecimento (Barret, 2003). Menor escolaridade relaciona-se com maior exposição a eventos adversos de vida, e desvantagens acumuladas como menores oportunidades ocupacionais, menor renda e mais doenças (Neri, 2007a). No presente estudo, renda pessoal não relacionou-se com sentir-se idoso, diferentemente das evidências de estudos internacionais (Barret, 2003). É possível que a pouca diferença socioeconômica da amostra do distrito pesquisado tenha gerado pouca variabilidade em comparação com as amostras dos estudos internacionais.

Indicadores negativos de saúde física e emocional relacionaram-se com sentir-se idoso. Os que perceberam sua saúde como muito ruim ou regular e os que foram classificados como frágeis identificaram-se como idosos. Presença de depressão e insatisfação com vida quando comparado a outros também foi frequente entre os que se sentem idosos. Por sua vez, menor depressão e maior satisfação comparada a outros se relacionou com a não identificação com a velhice. Keyes e Westerhof (2012), em conformidade com achados anteriores, encontraram que se sentir mais jovem do que a própria idade foi preditivo de melhor status de saúde física e mental, como menor risco para incapacidades, depressão e maior probabilidade de respostas adaptativas ao processo de envelhecimento.

O perfil dos indivíduos que tenderam a sentirem-se idosos foi dado pela ação conjunta de uma variável de natureza emocional e uma sociodemográfica, a saber, indivíduos moderadamente satisfeitos com a vida quando comparado a outros da mesma idade e de menor escolaridade. Considerando as influências da escolaridade já descritas, chama atenção o poder preditivo da variável satisfação com a vida comparada a outros. Teorias sociocognitivas, como a de comparação social (Festinger, 1954), por exemplo, sugerem que indivíduos constroem suas identidades etárias através da comparação das condições de sua vida (como saúde e bem-estar) com indivíduos da mesma idade ou com imagens generalizadas de pessoas pertencentes ao mesmo estrato etário. Assim, a adoção de uma identidade mais jovem ou discrepante pode ser interpretada como uma reinterpretação ativa da própria idade cronológica, pois manter uma identidade mais jovem requer um alto grau de percepção de controle e satisfação com a própria vida (Barret, 2003).

Ainda a respeito da saúde emocional, os resultados do presente estudo ratificam os estudos internacionais, como por exemplo, os dados do estudo MIDUS - Midlife Develo- pment in the United States (Barret, 2003). Assim como os resultados do estudo norteamericano, os indivíduos que não se identificam como idosos, ou seja, possuem identidades etárias mais jovens, relatam uma maior satisfação com a vida e mais afetos positivos.

Em linhas gerais, o presente estudo evidenciou que embora os idosos apontem uma idade para o início da velhice e que esta idade, em média, se assemelhe ao preconizado pelo Estado brasileiro, ainda prevalece certo afastamento ou não identificação, principalmente por parte de idosos mais jovens, com a categoria "idosos". Categorização etária da velhice associou-se com variáveis de diversas naturezas (sociodemográfica, saúde física, saúde mental e emocional) enquanto identificação etária associou-se com satisfação com a vida comparada a outros e escolaridade.

Algumas limitações metodológicas podem ser apontadas para futuros aperfeiçoamentos e investigações. Primeiro, destaca-se que, embora a amostra do presente estudo seja de base populacional e com idosos comunitários, peculiaridades do distrito pesquisado, tais como os baixos níveis educacionais e de renda, impeçam maiores generalizações a respeito das atitudes etárias de idosos no Brasil. Considera-se ainda a possibilidade de que futuras investigações levantem dados sobre a ocorrência de eventos de vida estressantes e incluam dados sobre a idade em que ocorreram transições importantes de vida. A inclusão de tais informações nas análises tem sido estimulada por estudiosos da gerontologia social e do paradigma do estresse na compreensão das avaliações pessoais da própria idade (Schafer \& Shippee, 2010).

Por fim, destaca-se a importância de mais estudos sobre a temática da categorização e identificação etária uma vez que podem auxiliar na compreensão do fenômeno e das repercussões do envelhecimento populacional brasileiro e examinar possíveis precursores de atitudes negativas e preconceitos em relação à idade, à velhice e ao envelhecimento. O conhecimento gerontológico e as políticas públicas voltadas aos idosos poderão usufruir desse saber, aperfeiçoando teorias e contextualizando práticas.

\section{Referências}

Abrams, D., \& Hogg, M. A. (2001). Collective identity: Group membership and self-conception. In M. A. Hogg \& R. S. Tindale (Eds.), Blackwell handbook of social psychology: Group processes (pp. 425-460). Oxford, England: Blackwell.

Abrams, D., Vauclair, C.-M., \& Swift, H. (2011). Predictors of attitudes to age across Europe (Research Report 735). London: Department for Work and Pensions. Retrieved from http://research.dwp.gov.uk/asd/asd5/rrs-index.asp

Almeida, O. P., \& Almeida, S. A. (1999). Confiabilidade da versão brasileira da Escala de Depressão em Geriatria (GDS) versão reduzida. Arquivos de Neuropsiquiatria, 57(2B), 421426. doi:10.1590/S0004-282X1999000300013

Barret, A. E. (2003). Socioeconomic status and age identity: The role of dimensions of health in the subjective construction of age. Journals of Gerontology: Social Sciences, 58(2), 101109. doi:1093/geronb/58.2.S101 
Batistoni, S. S. T., Prestes, S. M., Cachioni, M., Falcão, D. V. S., Lopes, A., Yassuda, M. S. \& Neri, A. L. (2015). Categorização e Identificação Etária em uma Amostra de Idosos Brasileiros Residentes na Comunidade.

Batistoni, S. S. T., \& Namba, C. S. (2010). Idade subjetiva e suas relações com o envelhecimento bem-sucedido. Psicologia em Estudo (Maringá), 15(4), 733-742. doi:10.1590/S141373722010000400009

Carstensen, L. L. (2006). The influence of a sense of time on human development. Science, 312(5782), 1913-1915. doi:10.1126/science. 1127488

Demakakos, P., Gjonca, E., \& Nazroo, J. (2007). Age identity, age perceptions, and health: Evidence from the English Longitudinal Study of Ageing. Annals of the New York Academy of Sciences, 1114, 279-287. doi:10.1196/annals.1396.021

Diener, E., Suh, M. E., Lucas, R. E., \& Smith, H. L. (1999). Subjective well-being: Three decades of progress. Psychological Bulletin, 125(2), 276-302. doi:10.1037/0033-2909.125.2.276

Ferrucci, L., Guralnik, J. M., Studenski, S., Fried, L. P., Cutler, G. B., \& Walston, A. J. D. (2004). Designing randomized, controlled trials aimed at preventing or delaying functional decline and disability in frail, older persons: A consensus report. Journal of American Geriatric, 52, 625-634. doi:10.1111/j.1532-5415.2004.52174.x

Festinger, L. (1954). A theory of social comparison processes. Human Relations, 7, 117-140. doi:10.1177/001872675400700202

Folstein, M. F., Folstein, S. E., \& McHugh, P. R. (1975). "Minimental state". A practical method for grading the cognitive state of patients for the clinician". Journal of Psychiatric Research, 12(3), 189-198.doi:10.1016/0022-3956(75)90026-6

Fried, L. P., Tangen, C. M., Walston, J., Newman, A. B., Hirsch, C., Gottdiener, J., ...McBurnie, M. A. (2001). Frailty in older adults: Evidence for a phenotype. Journals of Gerontolology, 56(A), 146-156. doi:10.1093/gerona/56.3.M146

Fundação Sistema Estadual de Análise de Dados. (2010). Município de São Paulo: População e estatísticas vitais. Recuperado em http://www.seade.gov.br/produtos/retratosdesp/view/

Keyes, C. L., \& Westerhof, G. J. (2012). Chronological and subjective age differences in flourishing mental health and major depressive episode. Aging and Mental Health, 16(1), 67-74. doi:10.1080/13607863.2011.596811

Levy, B. R., \& Myers, L. M. (2005). Relationship between respiratory mortality and self-perceptions of aging. Psychology and Health, 20, 553-564. doi:10.1080/14768320500066381

Levy, B. R., Slade, M. D., Kunkel, S. R., \& Kasl, S. V. (2002). Longevity increased by positive self-perceptions of aging. Journal of Personality and Social Psychology, 83, 261-270. doi:10.1037/0022-3514.83.2.261

Neri, A. L. (2007a). Qualidade de vida na velhice: Um enfoque multidisciplinar. Campinas, SP: Alínea.

Neri, A. L. (Ed.). (2007b). Idosos no Brasil: Vivências, desafios e expectativas na terceira idade. São Paulo, SP: Fundação Perseu Abramo.

Neri, A. L., Yassuda, M. S., Araújo, L. F., Eulálio, M. C., Cabral, B. E., de Siqueira M. E. C., ...Moura, J. G. A. (2013). Metodologia e perfil sociodemográfico, cognitivo e de fragilidade de idosos comunitários de sete cidades brasileiras: Estudo FIBRA. Cadernos de Saúde Pública, 29(4), 778-792. doi:10.1590/S0102-311X2013000400015

Rowe, J. W., Presidente, M. D., \& Kahn, R. L. (1996). Successful aging. The Gerontologist, 37(4), 433-440. doi:10.1093/ geront $/ 37.4 .433$

Sarkisian, C. A., Prohaska, T. R., Wong, M. D., Hirsch, S., \& Mangione, C. M. (2005). The relationship between expectations for aging and physical activity among older adults. Journal of General Internal Medicine, 20, 911-915. doi:10.1111/j.1525-1497.2005.0204.x
Schafer, M. H., \& Shippee, T. P. (2010). Age identity in context: Stress and the subjective side of aging. Social Psychology Quarterly, 73(3), 245-264. doi:10.1177/0190272510379751

Sheikh, R. L., \& Yesavage, J. A. (1986). Geriatric Depression Scale (GDS). Recent evidence and development of a shorter version. Clinical Gerontologist, 5, 165-173. doi:10.1300/ J018v05n01_09

Sneed, J. R., \& Whitbourne, S. K. (2003). Identity processing and selfconsciousness in middle and later adulthood. Journals of Gerontology: Psychological Sciences, 58, 313-319. doi:10.1093/geronb/58.6

Steverink, K., Westerhof, G., Bode, C., \& Dittmann-Kohli, F. (2001). The personal experience of aging, individual resources, and subjective well-being. Journal of Gerontology: Psychological Sciences, 56(6), 364-373. doi:10.1093/ geronb/56.6.P364

Sweiry, D., \& Willitts, M. (2001). Attitudes to age in Britains. House Research Departament for Work and Pensions, 7. Retrieved from http://research.dwp.gov.uk/asd/asd5/ihsindex.asp

Tajfel, H. (1981). Human groups and social categories. Cambridge, England: Cambridge University Press.

Westerhof, G. J. (2008). Age identity. In D. Carr (Ed.), Encyclopedia of the life course and human development: Vol. 3 (pp. 10-14). Farmington Hills, MI: Gale Cengage.

Westerhof, G. J., \& Barret, A. E. (2005). Age identity and subjective well-being: A comparison of the United States and Germany. Journal of Gerontology: Social Sciences, 60, 129-136. doi:10.1093/geronb/60.3.S129

Westerhof, G. J., Barret, A. E., \& Steverink, N. (2003). Forever young? A comparison of age identities in the United States and Germany. Aging, 25, 366-383. doi: $10.1177 / 0164027503025004002$
Recebido: $26 / 09 / 2013$

$1^{a}$ revisão: $12 / 05 / 2014$

Aceite final: 22/07/2014 